\title{
BMJ Open Primary healthcare professionals' perspective on vertical integration of healthcare system in China: a qualitative study
}

$\overline{\text { Shasha Yuan (D , }{ }^{1} \text { Fengmei Fan, }{ }^{2} \text { Joris van de Klundert, }{ }^{3,4} \text { Jeroen van Wijngaarden }{ }^{3}}$

To cite: Yuan S, Fan F, van de Klundert J, et al. Primary healthcare professionals' perspective on vertical integration of healthcare system in China: a qualitative study. BMJ Open 2022;12:e057063. doi:10.1136/ bmjopen-2021-057063

- Prepublication history and additional supplemental material for this paper are available online. To view these files, please visit the journal online (http://dx.doi.org/10.1136/ bmjopen-2021-057063).

Received 03 September 2021 Accepted 04 January 2022

Check for updates

(C) Author(s) (or their employer(s)) 2022. Re-use permitted under CC BY-NC. No commercial re-use. See rights and permissions. Published by BMJ.

${ }^{1}$ Institute of Medical Information \& Library, Chinese Academy of Medical Sciences \& Peking Union Medical Colleage, Beijing, China

${ }^{2}$ Peking University Huilongguan Clinical Medical School, Beijing, China

${ }^{3}$ Erasmus School of Health

Policy and Management,

Erasmus University Rotterdam, Rotterdam, Netherlands

${ }^{4}$ Prince Mohammad Bin

Salman College of Business \&

Entrepreneurship KAEC, King

Abdullah Economic City, Saudi Arabia

Correspondence to

Dr Jeroen van Wijngaarden;

vanwijngaarden@eshpm.eur.nl

\section{ABSTRACT}

Objective This study aims to present the perspectives of primary healthcare professionals (PHPs) on the impacts of implementation of vertical integration and on the underlying interprofessional collaboration process on achievement of the policy goals in China.

Design A qualitative study involving individual interview and group interview was conducted between 2017 and 2018.

Setting Primary healthcare institutions (PHIs) in five counties/districts of China.

Participants The major participants include 12 heads of PHIs (by 12 individual interviews) and 38 PHPs (by 12 group interviews). We also interviewed other stakeholders including 24 health policy-makers (by 5 group interviews) and 5 hospital leaders (by 5 individual interviews) for triangulation analysis.

Results Our study indicates that PHPs perceived vertical integration has resulted in improved professional competency, better care coordination and stronger capacity to satisfy patients' needs. The positive impacts have varied between integration types. Contributing factors for such progress are identified at administrative, organisational and service delivery levels. Other perceived effects are a loss of autonomy, increased workload and higher turnover of capable PHPs. Higher level hospitals play a dominant role in the interprofessional collaboration, particularly regarding shared goals, vision and leadership. These findings are different from the evidence in highincome countries. Incentive mechanisms and the balance of power with hospitals management are prominent design elements in the future.

Conclusions Our findings are particularly valuable for other countries with a fragmented health service system and low competency of PHPs as China's experience in integrated care provides a feasible path to strengthen primary care.

\section{INTRODUCTION}

The ageing of the population is rapidly accelerating worldwide. Elderly over 65 years accounted for $9.3 \%$ of the total world population in 2020 and will reach $17 \%$ by $2050 .{ }^{1}$ The growing proportion of elderly is associated with an increased incidence of multiple chronic medical conditions and followed by
Strengths and limitations of this study

- This is the first qualitative study to explore the primary healthcare professionals' perspective on the vertical integration of healthcare system in China.

- This study could greatly enrich academic evidence regarding the impacts of vertical integration which has been scant in undeveloped nations; and more importantly, provide practical values for other countries to strengthen primary healthcare.

- Different stakeholders involved in the reform of vertical integration in China were included to make the qualitative data more comprehensive.

- The interprofessional collaboration during vertical integration may have not been explicitly elicited as the interview protocol was not precisely guided by D'Amour model.

escalating disease burden. ${ }^{2}$ Primary healthcare is more accessible and more costeffective for home-based ageing population. ${ }^{3}$ However, it is challenging for many countries, and particularly for undeveloped nations like China, where healthcare systems are fragmented and the competency of primary health institutions (PHIs) is low. Providing people-centred and integrated healthcare has been regarded as a possible solution around the world. ${ }^{4}$ Among various integration models piloted in different countries, vertical integration, which involves coordination of services delivered by facilities at different levels (eg, PHIs and hospitals), is a major ingredient of integrated care. ${ }^{5}$ In China, the implementation of vertical integration has been a central feature of health reform to achieve the triple aims of reduced cost, improved quality and better population health. ${ }^{6}$

The health delivery system in China can be characterised as a three-tiered health service delivery network without mandatory gatekeeping mechanism. ${ }^{78}$ At the primary level, township health centres (THCs) in rural 
areas and community health centres (CHCs) in urban areas provide primary care, including both generalist care and public health services. ${ }^{9}$ The primary healthcare professionals (PHPs) working at these PHIs typically include general practitioners (GPs), physicians, nurses and public health workers. At the secondary and tertiary level, county/district hospitals (normally secondary level) and provincial/state-owned hospitals (normally tertiary level) usually provide comprehensive medical services. The financing mechanism differs between public hospitals and public PHIs in China. The government provides a very low proportion of funding to public hospitals, accounting only for around $9.6 \%$ of their total revenue in $2019,{ }^{10}$ while the public PHIs are fully funded. Because of a lack of systematic education and training for PHPs in the past, they are usually perceived as less competent than professionals at upper level hospitals. ${ }^{8}$ Data from the China Health Statistics in 2020 revealed that $48.6 \%$ of GPs in CHCs (urban areas) and $75.8 \%$ of those in THCs (rural areas) did not hold a bachelor's degree from a medical university. ${ }^{10}$ In contrast, $73.1 \%$ of doctors and physicians at hospitals had medicine bachelor degree and $20.8 \%$ of them finished postgraduate study. ${ }^{10}$ Thus, patients often bypass PHIs and directly turn to hospital care, which aggravates the crowdedness of hospitals and low efficiency of the health system in China. ${ }^{9}$ Therefore, strengthening primary healthcare has been prioritised in the new round of healthcare reforms since 2009 in China. Vertical integration is regarded as an important approach to achieve this goal. ${ }^{11}$

Since 2015, guided by national policy, local governments in China have piloted different vertically integrated care models. Some cities in east China already initiated the piloting of vertical integration prior to national implementation. Two types of models can be distinguished based on the integration of administrative, organisational and service delivery aspects: 'tight integration' and 'loose collaboration'. Under tight integration, the tertiary or secondary hospital usually takes charge, and is responsible for the management of vertical integration. Typical administrative strategies include establishing an independent management office and designating a deputy hospital director to be the head of integrated PHIs. A set of integration strategies at organisational and service levels is predetermined between the leading hospital and the participating PHIs. This may involve allocating more healthcare resources to PHIs, helping PHIs set up clinical departments with high demands, and conducting joint technical training for PHPs. ${ }^{12}$ In comparison, under loose collaboration, the integration strategies between participating hospitals and PHIs rarely happen at administrative and organisational level, but focus on the service level, often including technical assistance and skills training. ${ }^{13}$ Notably, the funding model of PHIs has not been changed. They are still financed by local governments.

The vertical integration reform in China is expected to achieve three main goals: improving professional competency of PHPs, enhancing coordination of primary and hospital care, and satisfying patients' health needs. The differences between primary care and hospital care, for instance, regarding service functions, financing mechanism and technical competence may complicate achievement of the policy goals. As PHPs are vital for strengthening primary care in China, it is relevant and worth studying their experiences with the vertical integration reform. Meanwhile, along with the implementation of vertical integration reform, collaboration of service delivery across different categories of service providers (called interprofessional collaboration) is a key component for providing integrated care. ${ }^{1415}$ However, the interprofessional collaboration process towards integrated care is often regarded as a 'black box' as little empirical evidence addressed how the partnership among different stakeholders evolve and are sustained. ${ }^{16} 17$ Therefore, besides the analysis of achieving the policy goals, it is also meaningful to explore the underlying interprofessional collaboration process under vertical integration in China.

The literature review shows that mixed evidence exists regarding the effects of vertical integration in healthcare systems. International research shows that results may vary depending on the context. Contradictory findings have been found in relation to health spending, health service utilisation and quality of care. ${ }^{18-20}$ A systematic review shows that vertical integration was associated with better quality, often measured as optimal care for specific conditions, but showed lower efficiency (or no efficiency improvement) as measured by utilisation, spending and prices. ${ }^{6}$ Meanwhile, some studies indicate that vertical integration increased health providers' market power and inappropriate referrals ${ }^{18}$ while professionals experienced a loss in practice autonomy. ${ }^{21}$ The few studies conducted on the impact of vertical integration on PHPs are from high-income countries.

Moreover, although research shows that interventions promoting interprofessional collaboration significantly improved health professionals' perceptions on quality of care and patient outcomes, ${ }^{22}$ the empirical evidence regarding the interprofessional collaboration process underlying vertical integration in primary care has been particularly scant. ${ }^{23}$ Therefore, it is essential to better understand how vertical integration can effectively be implemented among and between different professionals and organisations. ${ }^{24}$ The Four-Dimensional Model of Collaboration (D'Amour Model) is developed to model and understand collaboration structures. ${ }^{25}$ The model identifies four dimensions and ten indicators in the process of interprofessional collaboration in healthcare organisations. The four dimensions are shared goals and vision, internalisation, governance and formalisation (see online supplemental file 1). The model and the typology make it possible to analyse collaborating processes of health professionals and health institutions at different care levels during vertical integration. ${ }^{25}$ The model has been tested in different collaborative settings: in teams, between organisations, and in integrated healthcare networks. ${ }^{26-29}$ 
The objectives of this study are to explore the perspectives of PHPs on the impacts of implementation of vertical integration and the underlying interprofessional collaboration process on achievement of policy goals in China. Policy implications are further provided to enlighten future reform in China and shed some light on the countries expecting to develop integrated care.

\section{METHODS}

This study is of an explorative nature and follows a sequential approach of deductive and inductive steps. ${ }^{30}$ The methods and reporting follow the standards for reporting qualitative research (see online supplemental file 2). ${ }^{31}$

\section{Study setting}

Our study was conducted in Zhenjiang city in Jiangsu province (eastern part), Yichang city in Hubei province (middle part) and Chendu city in Sichuan province (western part). They were purposively selected by considering the type of vertical integration (tight integration vs loose collaboration) and geographical location (eastern vs western vs middle part and urban vs rural area). Table 1 shows the main characteristics of vertical integration for each setting and the sociodemographic information.

\section{Data collection}

The interviews were conducted between June 2017 and July 2018 (table 2). An interview protocol was developed based on the implementation and desired goals of vertical integration. Then, data were collected by individual interviews and group interviews based on the pre-determined interview guideline. The interviews were conducted faceto-face by two researchers (SY and FF) majoring in health policy in Chinese. Respondents were recruited until data saturation was reached. ${ }^{32}{ }^{33}$ Specifically, we conducted individual interviews for the heads of PHIs (around 1.5 hours) and group interviews for general PHPs consist of 1-2 GPs, 1-2 nurses and one public health worker (around 2 hours). The characteristics of participants is shown in online supplemental file 3 . The interview topics included general information on vertically integrated model piloted, implementation process, perceived effects and challenges during vertical integration (see online supplemental file 4 ).

To better understand the integration process at the administrative and organisational level, we also conducted interviews with other key stakeholders focusing on implementation processes at macro and meso level. A group interview (around 1.5 hours) was done with policy makers of health departments, which included one chief health officer at provincial level, one at city level and two or three at county level. Individual interviews (around 1 hour) were conducted with the directors of leading hospitals. Additionally, we also collected relevant documents concerning vertical integration (eg, local policy, working reports) as online supplemental materials.
Table 1 Characteristics of vertical integration and sociodemographic information

\begin{tabular}{|c|c|c|}
\hline $\begin{array}{l}\text { Study } \\
\text { setting }\end{array}$ & $\begin{array}{l}\text { Sociodemographic } \\
\text { information of sample } \\
\text { counties/districts }\end{array}$ & $\begin{array}{l}\text { Characteristics of } \\
\text { vertical integration }\end{array}$ \\
\hline \multirow[t]{2}{*}{$\begin{array}{l}\text { Zhenjiang } \\
\text { in Jiangsu } \\
\text { Province } \\
\text { (urban area) }\end{array}$} & $\begin{array}{l}\text { RZ district } \\
\text { Population (10 000): } \\
24.0 \\
\text { per capita disposable } \\
\text { income (RMB): } 45 \\
120 \\
\text { PHIs: } 8 \text { CHCs }\end{array}$ & $\begin{array}{l}\text { Tight integration } \\
\text { Time: } 2012 \\
8 \mathrm{CHCs} \text { covered }\end{array}$ \\
\hline & $\begin{array}{l}\text { JK district } \\
\text { Population (10 000): } \\
42.0 \\
\text { Per capita disposable } \\
\text { income (RMB): } 45 \\
693 \\
\text { - PHIs: } 6 \text { CHCs }\end{array}$ & $\begin{array}{l}\text { Loose collaboration } \\
\text { Time: } 2012 \\
6 \text { CHCs covered }\end{array}$ \\
\hline $\begin{array}{l}\text { Yichang in } \\
\text { Hubei } \\
\text { Province } \\
\text { (rural area) }\end{array}$ & $\begin{array}{l}\text { ZJ county } \\
\text { Population (10 000): } \\
\quad 47.8 \\
\text { Per capita disposable } \\
\text { income (RMB): } 17 \\
\quad 936 \\
\text { - PHIs: } 7 \text { THCs }\end{array}$ & $\begin{array}{l}\text { Tight integration } \\
\text { Time: } 2012-2013 \\
\text { - } 2 \text { THCs covered } \\
\text { Loose collaboration } \\
\text { Time: } 2016 \\
\text { 5 THCs covered }\end{array}$ \\
\hline \multirow[t]{2}{*}{$\begin{array}{l}\text { Chengdu } \\
\text { in Sichuan } \\
\text { Province } \\
\text { (urban and } \\
\text { rural areas) }\end{array}$} & $\begin{array}{l}\text { WH district } \\
\text { Population (10 000): } \\
64.7 \\
\text { Per capita disposable } \\
\text { income (RMB): } 42 \\
018 \\
\text { PHIs: } 10 \text { CHCs }\end{array}$ & $\begin{array}{l}\text { Loose collaboration } \\
\text { Time: } 2016 \\
10 \mathrm{CHCs} \\
\text { covered }\end{array}$ \\
\hline & $\begin{aligned} \text { XJ county } \\
\text { Population (10 000): } \\
31.7 \\
\text { Per capita disposable } \\
\text { income (RMB): } 20 \\
194 \\
\text { - PHIs:12 THCs }\end{aligned}$ & $\begin{array}{l}\text { Loose collaboration } \\
\text { Time: } 2016 \\
\text { 12 THCs covered }\end{array}$ \\
\hline
\end{tabular}

Data sources: sociodemographic information was from 2017 socioeconomic reports of sample counties/districts.

$\mathrm{CHCs}$, community health centres; PHIs, primary healthcare institutions; RZ, JK, ZJ, WH, XJ, abbreviations of the names of sample districts and counties.; THCs, township health centres.

\section{Data analysis}

The digital recordings were first transcribed and crossexamined by the first author (SY). Then, the materials in Chinese were translated by one professional translator and rechecked by SY. During the analyses, data were first categorised based on the codes from the interview protocol to identify if desired policy goals were reached and which contributing factors played a part. Then, the codes from D'Amour Model which encompasses 10 indicators in four dimensions were used to identify the interprofessional collaboration processes underlying the results above. Open labels (inductive) developed by SY, 
Table 2 The information of qualitative interviews

\begin{tabular}{|c|c|c|c|c|}
\hline & \multicolumn{2}{|l|}{ PHPs } & \multirow{2}{*}{$\begin{array}{l}\text { Policy-makers of health } \\
\text { departments (group } \\
\text { interview) }\end{array}$} & \multirow{2}{*}{$\begin{array}{l}\text { Leaders of participating } \\
\text { hospitals (individual } \\
\text { interview) }\end{array}$} \\
\hline & $\begin{array}{l}\text { Heads of PHIs } \\
\text { (individual interview) }\end{array}$ & $\begin{array}{l}\text { Other PHPs } \\
\text { (group interview) }\end{array}$ & & \\
\hline $\mathrm{RZ}$ district (Eastern) & 2 & $2(8)$ & $1(5)$ & 1 \\
\hline JK district (Eastern) & 2 & $2(6)$ & $1(5)$ & 1 \\
\hline XJ county (Western) & 2 & $2(6)$ & $1(5)$ & 1 \\
\hline \multicolumn{5}{|l|}{ Integration types } \\
\hline Tight integration & 4 & $6(20)$ & $2(9)$ & 2 \\
\hline Loose collaboration & 8 & $6(18)$ & $3(15)$ & 3 \\
\hline
\end{tabular}

The number in parenthesis shows the total number of participants interviewed in group interviews.

PHIs, primary healthcare institutions; PHPs, primary healthcare professionals; RZ, JK, ZJ, WH, XJ, abbreviation of the names of sample districts and counties.

FF and JvW were used to identify relevant themes that newly emerged at both stages. ${ }^{30}{ }^{34}$ Specifically, regarding the adaptation of D'Amour model in this study, we finally added incentive mechanism and kept dimensions of shared goals and vision, internalisation, formalisation and the indicator of leadership in the governance dimension. Triangulation in data collection and analysis was achieved by comparing the interviews of PHPs, with the interviews of other stakeholders and with the available documents. ${ }^{30}{ }^{33}$ MAXQDA2018 (VERBI Software, Bismarckstraße 10-12, 10625 Berlin) was used to process and code the data.

\section{Patient and public involvement}

No patient or public were involved. The participants were not invited to contribute to the analysing or writing of the paper except the interviews.

\section{RESULTS}

Tables 3 and 4 summarise PHPs' perceptions on achieving the desired goals of vertical integration and the underlying process of interprofessional collaboration.

\section{PHPs' perceptions on achieving desired policy goals and contributing factors \\ Professional competency improvement}

Positive effect of vertical integration on professional competency were commonly reported by PHPs. Existing skills and knowledge were improved and new skills and knowledge were developed, according to most respondents. PHPs under tight integration, particularly GPs, reported more benefits from vertical integration than those under loose integration. Factors at both the service delivery level and the organisational level contributed.

At the service delivery level, joint training programmes were generally provided based on the needs of PHIs and the available expertise of the hospitals. The technical trainings were free for PHIs to increase PHPs' participation. Training activities about diagnosis and treatment guidelines for common diseases were frequently mentioned by PHPs. Common training contents included (1) rational use and management of antibiotic drugs, (2) practice guidelines of hypertension, diabetes and stroke and (3) outpatient prescription review and assessment. Training took the shape of internships of PHPs in the hospital (1 month to half a year) or hospital experts visiting PHIs at a regular basis to treat patients and provide training. For example, it is typically reflected that 'The training between leading hospital and our THC was very flexible. The hospital was ready to help as long as we make clear requests for assistance. The time was flexible like 3 months, 6 months or 1 year. Such training was beneficial for both us and our department'.

At the organisational level, under the support of leading hospitals and local governments, a new clinical department was usually established (tight integration) to better satisfy patient needs. For example, in RZ district, new rehabilitation wards were built at the integrated PHI to meet the rehabilitation needs of local residents. The leading hospital assigned professional teams to assist management staff and arrange skills training activities. The final goal was to achieve quality homogeneity and maximally stimulate downward referral. The management and clinical skills of PHPS were perceived to have greatly improved by the training provided. 'The rehabilitation department of the leading hospital is very strong. After we integrated our service delivery, the hospital provided great help to construct rehabilitation wards at our CHC and provide intensive trainings for us. Now, we are capable of receiving the patients (such as stroke) referred to us' (responded by one GP). 
Table 3 Summary of PHPs' perception on contributing factors towards desired policy goals

\begin{tabular}{|c|c|c|c|}
\hline $\begin{array}{l}\text { Policy goals } \\
\text { of vertical } \\
\text { integration }\end{array}$ & \multicolumn{3}{|l|}{ Contributing factors } \\
\hline $\begin{array}{l}\text { Professional } \\
\text { competency } \\
\text { improvement }\end{array}$ & & $\begin{array}{l}\text { Establishing new clinical } \\
\text { departments with high health } \\
\text { needs at PHIs, typically } \\
\text { including departments of } \\
\text { Chinese medicine therapy, } \\
\text { laboratory tests and } \\
\text { rehabilitation } \\
\text { Common for tight integration }\end{array}$ & $\begin{array}{l}\text { Professional training activities for } \\
\text { PHPs existed for both types } \\
\text { Tight integration: The training } \\
\text { activities were conducted in a more } \\
\text { scheduled way, including clear training } \\
\text { objectives, frequency of hospital } \\
\text { experts to PHIs, priority clinical skills } \\
\text { trained, etc. } \\
\text { Loose collaboration: The training } \\
\text { activities were relatively flexible; } \\
\text { normally based on PHIs' requirement }\end{array}$ \\
\hline $\begin{array}{l}\text { Satisfying } \\
\text { patients' health } \\
\text { needs }\end{array}$ & & $\begin{array}{l}\text { Image centre located in the } \\
\text { higher level hospital where } \\
\text { hospital specialists provide } \\
\text { diagnosis for the upload images } \\
\text { from PHIs } \\
\text { Guided by local government; } \\
\text { common for both types of } \\
\text { integration }\end{array}$ & $\begin{array}{l}\text { Higher access to specialist services, } \\
\text { examination services and timely } \\
\text { upreferral at PHIs } \\
\text { Improving financial access by paying } \\
\text { based on PHI fee schedule }\end{array}$ \\
\hline
\end{tabular}

PHIs, primary healthcare institutions; PHPs, primary healthcare professionals.

\section{Care coordination enhancement}

It was widely reported that the coordination of primary care and hospital care was enhanced through vertical integration, particularly relating to referral from PHIs to participating hospitals. The reform provided a feasible solution for the PHIs to coordinate collaboration with the hospitals, which was fragmented in the past. Dual referral systems between participating PHIs and hospitals were established. However, the effects varied greatly in reality as referral was not mandatory for accessing higher level facilities.

At the administrative level, the most distinct characteristic between tight integration and loose collaboration was related to the role of hospital in the management of PHIs. It is common for tight integration to designate a deputy hospital president to be the head of the integrated PHIs. The designated head would work full time at and with the PHIs, while remaining an employee of the hospital. Such administrative arrangements were beneficial for strengthening organisational coordination between hospitals and PHIs as members of the same integrated care network. The PHPs felt that under this structure their facilities were more tightly integrated with the hospital, 'The head of our
THC is from Renmin County Hospital and he was the deputy president of the hospital. We are more like a subdivision of the hospital. It is convenient for us to refer patients to higher level hospitals and require for joint consultations for our patients.' Besides, the designated heads were perceived to have better management skills, which facilitated the development of PHIs.

At the organisational level, special offices and persons responsible for integration (such as dual referral) were put in place. They were usually full-time persons under tight integration while part-time persons were more common for loose collaboration. Interagency cooperation was mainly achieved through a newly established 'green channel' (ie, unobstructed channel) for case referral between PHIs and participating hospitals. Standard processes for dual referral were set up by the aforementioned special integrated offices. Patients' willingness also needed to be respected as referral was not mandatory. It was also frequently mentioned that local government paid special attention to electronic information sharing for dual referral and service provision. In practice, PHPs were prone to contact their counterparts in the corresponding hospital department directly, instead of using the information system. 
Table 4 The process of interprofessional collaboration during vertical integration in China

\begin{tabular}{|c|c|c|}
\hline Collaboration & Practices of vertical integration in China & ation tynes \\
\hline $\begin{array}{l}\text { Shared goals } \\
\text { and vision }\end{array}$ & $\begin{array}{l}\text { Goals: Clearly set by national government; PHIs acted as 'receiver' of } \\
\text { hospitals' integration strategies } \\
\text { Client-centred orientation versus other allegiances: Client-centred } \\
\text { orientation was clearly shown as satisfying patients' needs at the setting of } \\
\text { desired policy goals of vertical integration. }\end{array}$ & $\begin{array}{l}\text { The leading hospital played a } \\
\text { dominant role; more obvious } \\
\text { in tight integration. }\end{array}$ \\
\hline Internalisation & $\begin{array}{l}\text { Mutual acquaintanceship: The familiarisation processes occurred at training } \\
\text { activities, professional support and communication through personal phone } \\
\text { calls, we-chat groups and QQ groups. } \\
\text { - Trust: More reflected at trust from PHPs to hospital experts }\end{array}$ & $\begin{array}{l}\text { Higher intensity in tight } \\
\text { integration }\end{array}$ \\
\hline \multirow[t]{4}{*}{ Governance } & $\begin{array}{l}\text { Centrality: Guided by national government (eg, national policy); designed } \\
\text { and led by local government (eg, detailed implementation plan). }\end{array}$ & Almost the same \\
\hline & $\begin{array}{l}\text { Leadership: Usually led by the hospitals; under tight integration, the hospital } \\
\text { leadership occurred at administrative level (eg, designating head of PHI), } \\
\text { organisational level (eg, full-time persons responsible for integrated issues) } \\
\text { and service level (eg, training sessions); under loose collaboration, the } \\
\text { leadership was mainly reflected at service level (eg, training sessions). }\end{array}$ & $\begin{array}{l}\text { PHPs under tight integration } \\
\text { perceived higher power of the } \\
\text { hospital leadership, therefore, } \\
\text { felt more threatened towards } \\
\text { losing autonomy }\end{array}$ \\
\hline & - Support for innovation: newly developed clinical skills such as rehabilitation. & $\begin{array}{l}\text { More frequently seen in tight } \\
\text { integration }\end{array}$ \\
\hline & $\begin{array}{l}\text { Connectivity: technical exchanges, teamwork for patients' care and dual } \\
\text { referral established during vertical integration between PHIs and hospitals. }\end{array}$ & $\begin{array}{l}\text { Higher intensity in tight } \\
\text { integration }\end{array}$ \\
\hline Formalisation & $\begin{array}{l}\text { Formalisation tools: interorganisational agreements signed between PHIs } \\
\text { and hospitals; different professional protocols formed (mostly by hospitals). } \\
\text { Information exchange: usually based on local information system, typically } \\
\text { including image centre and remote medical treatment centre; located at } \\
\text { hospital. }\end{array}$ & Almost the same \\
\hline
\end{tabular}

PHIs, primary healthcare institutions; PHPs, primary healthcare professionals.

At the service delivery level, mutual acquaintance between PHPs and hospital experts was formed gradually through regular personal interaction during the training sessions, stimulating trust, mutual help and continuous communication through personal phone calls, we-chat groups and QQgroups (the two popular mobile communication software used in China). Respondents talked about increased teamwork. For example, one GP mentioned ' $I$ discuss patients' information including syndrome, diagnosis, examination results and treatment effects with the physicians of the integrated hospital by $Q Q$ group. I was trained in his department for half a year so we are familiar with each other. They [the hospital physicians] gave me timely advises. It sounds like PHPs have an expert team working together.'

\section{Satisfying patients' health needs}

The benefits vertical integration brought for patients were frequently reported by PHPs. These benefits were closely related with better professional competency of PHPs and better care coordination between PHIs and hospitals as mentioned above. These benefits include better access to hospital expert services, examination convenience and a relatively smooth process of hospital referral.

At the service delivery level, it was obvious for all respondents that patients had much easier access to hospital specialists, as they would directly work together on a regular basis with PHIs in the community. PHPs also found it much easier to arrange additional examinations such as imaging scans for the patients. In the past, it was considered more difficult to provide examination services due to lack of capable health professionals at PHIs. The patients had to visit hospitals for obtaining such services. After vertical integration, PHPs now could upload all the images to the image centre at hospital where specialists were available to deal with them and provide feedback. Easier access to hospital experts and examinations were considered particularly convenient for the elderly population. Patients also had lower financial burden since all expert services and examinations were provided by PHIs where charges for the same service are lower compared with higher level hospitals.

The referral channel opened by tertiary hospitals was particularly useful according to the interviewees. In the past, it was difficult for the patients to register with those clinical departments renowned for distinguished skills at tertiary hospital. Under vertical integration, the leading hospitals were required to reserve certain outpatient appointments and inpatient beds for the integrated PHIs. Therefore, it greatly saved the time of patients 
and improved their treatment experiences, as reflected by PHPs, 'They (outpatient appointments and inpatient beds at hospital) are scarce resources especially for famous specialists. We could help patients get timely treatment as the hospital has already reserved them for us.' 'It was believed that some patients visited PHIs hoping to obtain the hospital outpatient appointment, rather than to seek health services from PHPs.

\section{Additional effects of vertical integration perceived by PHPs \\ Risk of losing autonomy}

The changes of autonomy were mostly reported by the heads of PHIs and less by regular PHPs. Concerns about losing management autonomy were more common for those under tight integration. During vertical integration, annual performance evaluation of PHIs was now conducted by the participating hospitals instead of by the local health department. The hospitals also took the responsibility of designating and recommending heads of the integrated PHIs. Some heads of PHIs perceived to have become affiliated with the hospital and to have lost their independency. The impact of integration on autonomy was more frequently discussed by the PHIs with higher competencies, where the leaders preferred loose collaboration over tight integration.

\section{Increasing workload}

The increasing workload was mainly reported by GPs and nurses under tight integration and particularly for those PHIs with newly established departments (such as physiotherapy department of traditional Chinese medicine and rehabilitation wards). In contrast, the interviewees under loose collaboration did not report obvious changes of workload resulting from vertical integration. They were more prone to consider increases in patient volume to be caused by their own development.

\section{Turnover of capable PHPs}

Turnover here refers to recruitment of more competent PHPs by higher level hospitals. The phenomenon is closely related to improved professional competency of PHPs and better care coordination with participating hospitals during vertical integration. For the PHPs, such recruitment provided development opportunities and job opportunities. For instance, the hospitals usually provided a better platform to advance professional skills. Promotion was also perceived to be relatively faster in hospitals. It is interesting to find that the heads of PHIs and other PHPs held different opinions on this turnover. The retention of skilled GPs and nurses is challenging for PHIs due to low salary and status. The heads of PHIs found that the reform of vertical integration (particularly tight integration) increased the possibility of the most capable PHPs flowing to the hospitals, especially for those county hospitals that had recruitment difficulties themselves.
Interprofessional collaborating process underlying vertical integration

Shared goals and vision

The policy goals of vertical integration reform were clearly set by national government, as described above, that is, improving professional competency of PHPs, enhancing coordination of primary healthcare and hospital care, and satisfying patient health needs. Towards this vision, each integrated care network implemented a collection of administrative, organisational and service interventions. The above analysis regarding the achievement of desired policy goals indicated that the hospitals and PHIs under vertical integration have been working together and partly achieved them. However, because of the higher status of hospitals in the Chinese culture, the PHIs acted as 'receiver' of participating hospitals' integration strategies. They were not considered as equals and the hospitals were prone to implement the reform according to their own interest. For example, there was almost no training targeting public health service, which forms one of the two major service functions of PHIs in China. Accordingly, the interviewed public health worker reported the least positive experiences towards vertical integration. Further, the clinical trainings were also largely dependent on the willingness and abilities of the hospitals rather than on demand of PHIs.

\section{Internalisation}

As described by D'Amour model, internalisation was shown through mutual acquaintanceship and trust. The familiarisation processes occurred at training activities, professional support and communication through personal phone calls, we-chat groups and QQ groups. Since PHPs under tight integration had more chance to participate in training activities, they felt more acquainted with hospital specialists compared with those under loose collaboration. Moreover, mutual acquaintance was reported to have improved PHPs' professional confidence. PHPs perceived that mutual acquaintance enhanced the process of upward referral and joint consultation by receiving timely support from hospital specialists when treating patients.

Regarding mutual trust, PHPs reported to trust hospital specialists as they would seek and follow professional advice from hospital specialists when necessary. An indicator of increased trust of hospital specialists in PHPs from the RZ district is that, 'Now most of our patients in the new rehabilitation wards are referred from the participating hospital. We gradually gain the trust of both hospital specialists and patients'. PHPs also perceived that the close relationship and mentorship established with hospital professionals promoted patient trust in PHPs.

\section{Governance}

Compared with 'Centrality', 'Support for innovation' and 'Connectivity', 'Leadership' was more frequently talked about by the heads of PHIs. The leadership role was usually held by the hospitals (as relates to the perceived 
lack of autonomy by the heads of PHIs mentioned above). Under tight integration, the hospital leadership took shape at the administrative level (eg, designating a head of PHI), the organisational level (eg, full time persons responsible for integrated issues) and at the service level. In comparison, the leadership under loose collaboration was mainly reflected at the service level (eg, training sessions). Therefore, the negative impact of hospital leadership might have been more commonly perceived by heads of the PHIs under tight integration and by PHIs already having stronger competencies before the reform: 'It is not suitable to implement one-size policy for all. The competency of my institution is relatively good even without support of the hospitals. I am also capable of managing our CHC. I think the integrated hospital should not intervene too much in our business operation'.

\section{Formalisation}

Different types of formalisation tools are found during vertical integration. First, the agreements were usually signed between PHIs and hospitals under the same vertically integrated models. In WH district, the contracts were signed between local government (representing PHIs in $\mathrm{WH}$ ) and participating hospitals. These agreements regulated the mutual responsibilities and the duration of vertical integration. Second, different protocols were also formed, typically including dual referral, treatment guidelines for common diseases, and the uniform drug lists. These protocols were mostly seen by the respondents as helpful to improve the quality of primary care; 'The hospital specialists helped improve the clinical pathways of 151 common disease encountered at PHIs so we could practice in a more standard way. It could also serve as the basis of dual referral. The patients with the disease outside the catalogue were more likely to be referred to the hospital'. The information exchange was based on local information systems, and included images and telemedicine.

\section{Incentive mechanism}

Incentive mechanisms are not included as a key indicator in D'Amour model but frequently reported by the PHPs. At present, for participating hospitals, the technical trainings and the imaging services they provided were free for PHIs. Hospitals appeared to be largely motivated by increased administrative power. Besides, higher level hospitals hoped that such arrangement will help them secure patients in the future if gatekeeping by primary care facilities becomes mandatory. Lack of financial incentives during vertical integration was regarded as one of the most challenging issues according to PHPs, which made them uncertain of the sustainability of vertical integration.

Meanwhile, PHPs under vertical integration also lack financial incentives. The growth of medical revenue at PHIs from increases in outpatient visits and inpatient stays resulting from vertical integration did not lead to higher salary for PHPs. The salary system of PHPs in China usually includes a large fixed part and a capped flexible part which relates to PHI revenue. One GP under tight integration mentioned, 'We are under-paid actually. The increasing medical revenue after vertical integration did not change this situation. There is a ceiling for our salary set by local government and I think it is not suitable for our current workload.'

\section{DISCUSSION}

Based on PHPs' perceptions, our study indicates that the vertical integration reform in healthcare system of China partly achieved its desired policy goals as shown by improved professional competency, better care coordination, and stronger capacity to satisfy patients' needs. Additional perceived effects of vertical integration revealed by our study are a loss of autonomy, increasing workload, and turnover of capable PHPs. The analysis of underlying interprofessional collaboration indicates that higher level hospitals were more likely to play a dominant role as a result of vertical integration, particularly regarding the shared goals, vision and leadership. The perceived impacts of vertical integration varied between tight integration and loose collaboration.

First, our findings indicate that the PHPs under tight integration were more prone to report improved professional competency and better care coordination compared with those under loose collaboration. This forms qualitative empirical evidence for the debate on the selection of integration type in practice. The difference in additionally perceived positive changes for tight integration may result from administrative and organisational arrangements which promoted more effective training activities, technical communications and newly developed skills at service level. In the meantime, our study also finds that tight integration may lead to a loss of autonomy and turnover of capable PHPs. These negatives were rarely reported under loose collaboration. Such concerns of reduced autonomy were also expressed by physicians from Accountable Care Organisation in the USA. ${ }^{21}$ The difference is that loss of autonomy was mostly reported by the heads of PHIs in China instead of normal PHPs while it was a great concern of GPs and physicians in America. In China, PHPs appeared to have no or few objections to comply with technical advice from hospital professionals. ${ }^{8}$ The difference in professional competency in comparison to hospital specialists may explain why PHPs rarely perceived a threat to their professional autonomy, thus facilitating the collaboration process.

Additionally, the longer duration of tight integration in the sample counties may also contribute to different perspectives of PHPs. In our study, the reform of tight integration in RZ district and ZJ county has been initiated since 2012 prior to the national reform in 2015. It is also 4 years earlier than most PHIs covered by loose collaboration. The local governments were able to implement relatively more policies and make them more adaptable. Therefore, the policy effects of tight integration were more likely to be shown and captured by PHPs. 
Second, the interprofessional collaboration shown during vertical integration in China was greatly influenced by the Chinese culture and characteristics of China's health system. PHPs' social and professional status is generally perceived lower compared with hospital specialists in China, due to lack of high-quality training and education systems. ${ }^{8}$ The majority of PHPs received their medical training no more than 4 years after the completion of secondary school. It is totally different from GPs and physicians in developed countries, where they are more equivalent in both education background and professional status. Consequently, PHP's attitude towards professional autonomy in China is not as highincome countries. Therefore, PHPs in China are more likely to follow the guidance of hospital professionals and more easily subscribe to shared goals and leadership of the hospital and specialists during interprofessional collaboration. In contrast, empirical evidence from highincome countries has revealed that achieving mutual goals is always challenging and takes considerable time from health professionals at different levels. ${ }^{23} 2426$

Third, the lack of incentive mechanisms to ensure the sustainability of vertical integration has become one of the most challenging issues for future reform in China. Our study indicates the vital role of incentive mechanisms (particularly financial incentive) to strengthen interprofessional collaboration between PHIs and hospitals. These incentives are not included in the model of D'Amour. Evidence from other countries also point out the importance of financial incentives when implementing integrated care. For instance, in Germany, financial incentives, which were from the savings compared with risk-adjusted standardised costs, were provided for both sickness funds (insurers) and providers to proceed integrated care between ambulatory care providers and hospitals. ${ }^{35}$ In the USA, Accountable Care Organisations were rewarded with shared savings bonuses if spending is sufficiently below benchmarks (spending target). ${ }^{36}$ Recently, some counties in China initiated bundled payment reform for participating PHIs and hospitals ${ }^{37} 38$ but it is still too early to decide its effects. We recommend the design of suitable incentive mechanism for interprofessional collaboration as an important area for future research and to advance the sustainability of vertical integration.

Lastly, let us emphasise the perceived positive role of vertical integration on strengthening primary healthcares in China. Moreover, it merits particular mentioning that PHPs reported benefits to patients under both types of integration. Patient-centred integrated care was at the heart of integrative strategies proposed by WHO in $2015 .{ }^{4}$ They contribute to retaining patients at primary care level and to strengthening the gatekeeping role of PHPs. These benefits of vertical integration to primary healthcare are particularly valuable for low and middle income countries that are also confronted with a traditionally weaker primary care infrastructure and limited funding. The piloting experience of vertical integration in China may provide a feasible path for them to strengthen primary healthcare, which was missing in scientific literature and urgently needed in practice.

Our study also comes with limitations. First, it particularly focused on the perception of PHPs under vertical integration. Online supplemental data from policy documents and other stakeholders including policy makers of health departments at different levels and participating hospital directors were collected and used for data triangulation analysis. However, the findings would be more comprehensive if patient perspective had been included. Second, the interview protocol was mostly organised around the perception of PHPs on the achievement of policy desired goals. Therefore, the perceptions of interprofessional collaboration dimensions of D'Amour model have not been explicitly elicited but are derived from qualitative analysis. Future quantitative evaluation studies based on D'Amour Model and the findings of this study can advance understanding in this domain.

\section{Policy implications}

Three policy implications are provided to enlighten the future reform in China, and moreover, to shed some new light on other countries aiming to develop integrated care. First, under the context of China, vertical integration between PHIs and hospitals (particularly tight integration) probably stimulated the improvement of professional competency and coordination of primary care and hospital care. However, further targeting strategies are needed to ensure the management and professional autonomy of PHIs and retain qualified and capable PHPs. Second, appropriate incentive mechanisms (particularly financial incentives) should be developed in the future reform to advance the sustainability of vertical integration. Health provider payment reform may become a possible solution to this issue. Finally, the perceived impacts of vertical integration are closely related with the characteristics of China's health system and governance and cultural background. The unique features particularly worthy to be concluded include the historical fragmentation between primary care and hospital care, a relatively larger gap between health professionals at different care levels and all the health institutions under the supervision of central and local governments. Therefore, cautious attitude need to be kept when other countries develop their own vertical integration strategies based on China's experience.

\section{CONCLUSION}

Our study indicates that vertical integration played a positive role in achieving desired policy goals concerning primary healthcare from the perspectives of PHPs in China, particularly regarding professional competency improvement, better care coordination and satisfaction of patient needs. The benefits were more prominent under tight integration than under loose collaboration. Furthermore, the study reveals that higher level hospitals actually played a dominant role in the interprofessional collaboration, particularly regarding shared goals, vision 
and leadership, which is different from the evidence in high-income countries. Our findings are particularly valuable for other countries with a fragmented health service system and similar characteristics in governance and cultural background as China's experience in integrated care provides a feasible path to strengthen primary care.

Contributors SY conceptualised study design, analysed the data and drafted the manuscript; FF participated data collection and analysis; JvW and JvdK performed critical revision of article for important intellectual content. JvW is responsible for the overall content as the guarantor.

Funding The authors have not declared a specific grant for this research from any funding agency in the public, commercial or not-for-profit sectors.

Competing interests None declared.

Patient consent for publication Not applicable.

Ethics approval This study involves human participants and was approved by Institute of Medical Information and Library Human Research Ethics Committee (HREC); Ref. No. IMICAMS/01/19/HREC.

Provenance and peer review Not commissioned; externally peer reviewed.

Data availability statement № data are available. Not applicable.

Supplemental material This content has been supplied by the author(s). It has not been vetted by BMJ Publishing Group Limited (BMJ) and may not have been peer-reviewed. Any opinions or recommendations discussed are solely those of the author(s) and are not endorsed by BMJ. BMJ disclaims all liability and responsibility arising from any reliance placed on the content. Where the content includes any translated material, BMJ does not warrant the accuracy and reliability of the translations (including but not limited to local regulations, clinical guidelines, terminology, drug names and drug dosages), and is not responsible for any error and/or omissions arising from translation and adaptation or otherwise.

Open access This is an open access article distributed in accordance with the Creative Commons Attribution Non Commercial (CC BY-NC 4.0) license, which permits others to distribute, remix, adapt, build upon this work non-commercially, and license their derivative works on different terms, provided the original work is properly cited, appropriate credit is given, any changes made indicated, and the use is non-commercial. See: http://creativecommons.org/licenses/by-nc/4.0/.

ORCID iD

Shasha Yuan http://orcid.org/0000-0002-5091-6688

\section{REFERENCES}

1 United Nation. World population prospects, 2019. Available: https:// population.un.org/wpp/ [Accessed 9 Mar 2021].

2 Goodwin N, Dixon A, Anderson G. Providing integrated care for older people with complex needs lessons from seven international case studies. London: The King's Fund, 2014.

3 Langlois EV, McKenzie A, Schneider $\mathrm{H}$, et al. Measures to strengthen primary health-care systems in low- and middle-income countries. Bull World Health Organ 2020;98:781-91.

4 World Health Organization. WHO global strategy on people-centred and integrated health services. Geneva: World Health Organization, 2015.

5 Kodner DL. All together now: a conceptual exploration of integrated care. Healthc Q 2009;13 Spec No:6-15.

6 Machta RM, Maurer KA, Jones DJ, et al. A systematic review of vertical integration and quality of care, efficiency, and patientcentered outcomes. Health Care Manage Rev 2019;44:159-73.

7 Liu Y, Kong Q, Yuan S, et al. Factors influencing choice of health system access level in China: a systematic review. PLoS One 2018;13:e0201887.

8 Yuan S, Wang F, Li X, et al. Facilitators and barriers to implement the family doctor contracting services in China: findings from a qualitative study. BMJ Open 2019;9:e032444.

9 Li X, Krumholz HM, Yip W, et al. Quality of primary health care in China: challenges and recommendations. Lancet 2020;395:1802-12.

10 National Health Commission. The 2019 statistics Yearbook of national health. Beijing: China Union Medical University Pres, 2020.
11 Shi L, Makinen M, Lee D-C, et al. Integrated care delivery and health care seeking by chronically-ill patients - a case-control study of rural Henan Province, China. Int J Equity Health 2015;14:1-15.

12 Wang X, Birch S, Ma H, et al. The structure and effectiveness of health systems: exploring the impact of system integration in rural China. Int J Integr Care 2016;16:6.

13 Yuan S, Wang F, Zhao Y, et al. Assessing perceived quality of primary care under hospital-township health centre integration: a cross-sectional study in China. Int $\mathrm{J}$ Health Plann Manage 2020;35:e196-209.

14 D'Amour D, Ferrada-Videla M, San Martin Rodriguez L, et al. The conceptual basis for interprofessional collaboration: core concepts and theoretical frameworks. $J$ Interprof Care 2005;19 Suppl 1:116-31.

15 Valentijn PP, Schepman SM, Opheij W, et al. Understanding integrated care: a comprehensive conceptual framework based on the integrative functions of primary care. Int $J$ Integr Care 2013;13:e010.

16 Axelsson R, Axelsson SB. Integration and collaboration in public health--a conceptual framework. Int $J$ Health Plann Manage 2006;21:75-88.

17 de Stampa M, Vedel I, Bergman H, et al. Opening the black box of clinical collaboration in integrated care models for frail, elderly patients. Gerontologist 2013;53:313-25.

18 Baker LC, Bundorf MK, Kessler DP. Vertical integration: Hospital ownership of physician practices is associated with higher prices and spending. Health Aff 2014;33:756-63.

19 Post B, Buchmueller T, Ryan AM. Vertical integration of hospitals and physicians: economic theory and empirical evidence on spending and quality. Med Care Res Rev 2018;75:399-433.

20 Neprash HT. Vertical integration likely increases spending, but does it also improve quality of care? J Gen Intern Med 2020;35:630-2.

21 Berenson RA. A Physician's Perspective On Vertical Integration. Health Aff 2017;36:1585-90.

22 Zwarenstein M, Reeves S, Perrier L. Effectiveness of pre-licensure interprofessional education and post-licensure collaborative interventions. J Interprof Care 2005;19 Suppl 1:148-65.

23 Valentijn PP, Vrijhoef HJM, Ruwaard D, et al. Exploring the success of an integrated primary care partnership: a longitudinal study of collaboration processes. BMC Health Serv Res 2015;15:32.

24 Valentijn PP, Ruwaard D, Vrijhoef HJM, et al. Collaboration processes and perceived effectiveness of integrated care projects in primary care: a longitudinal mixed-methods study. BMC Health Serv Res 2015; 15:463.

25 D'Amour D, Goulet L, Labadie J-F, et al. A model and typology of collaboration between professionals in healthcare organizations. BMC Health Serv Res 2008;8:1-14.

26 Nuño-Solinís R, Zabalegui IB, Rodríguez LSM, et al. Does interprofessional collaboration between care levels improve following the creation of an integrated delivery organisation? the Bidasoa case in the Basque country. Int $J$ Integr Care 2013;13:e030.

27 Polanco NT, Zabalegui IB, Irazusta IP, et al. Building integrated care systems: a case study of Bidasoa integrated health organisation. Int J Integr Care 2015;15:e026.

28 Tang W, Zhang Y, Zhang L. Rebuild and evaluate the effect of multiinstitutional collaboration model in healthcare system integration reform: evidence from a community-based rural quasi-experiment. Chinese Journal of Health Policy 2016;9:6-11.

29 Yuan S, Jia M, Wang F. Comparatively analyzing the collaborating mechanism of primary healthcare institutions and comprehensive hospital in different medical integrated models. The Chinese Health Service Management 2019;36:81-3.

30 Azungah T. Qualitative research: deductive and inductive approaches to data analysis. Qualitative Research Journal 2018;18:383-400.

31 O'Brien BC, Harris IB, Beckman TJ, et al. Standards for reporting qualitative research: a synthesis of recommendations. Acad Med 2014;89:1245-51.

32 Saunders B, Sim J, Kingstone T, et al. Saturation in qualitative research: exploring its conceptualization and operationalization. Qual Quant 2018;52:1893-907.

33 Green J, Thorogood N. Qualitative methods for health research (introducing qualitative methods series). 4th ed. London: SAGE, 2018.

34 Klundert Jvande, de Korne D, Yuan S, et al. 'Hybrid' top down bottom up health system innovation in rural China: a qualitative analysis. PLoS One 2020;15:e0239307.

35 Busse R, Stahl J. Integrated care experiences and outcomes in Germany, the Netherlands, and England. Health Aff 2014;33:1549-58. 
36 McWilliams JM, Hatfield LA, Landon BE, et al. Medicare spending after 3 years of the Medicare shared savings program. N Engl J Med 2018;379:1139-49.

37 Yuan S, Yong Z, Gao H. Quality assessment of primary healthcare institutions entrusted to private hospitals. Chin J Hosp Admin 2018;34:273-8.
38 Chen C. Investigation on the implementation effect of County medical community in Tongren City. Guizhouzz: Guizhou Medica University, 2019. 